\title{
Model structure simplification of a biological reactor
}

\author{
Anca Maria Nagy*, Gilles Mourot*, Benoît Marx* \\ Georges Schutz**, José Ragot*

\begin{abstract}
* Centre de Recherche en Automatique de Nancy, Nancy Université, Institut National Polytechnique de Lorraine, 2, avenue de la Forêt de Haye, 54516 Vandoeuvre-lès-Nancy Cedex France

** Centre de Recherche Public "Henri Tudor", Laboratoire de

Technologies Industrielles et Matériaux, 29, Avenue John F.Kennedy, L-1855 Luxembourg-Kirchberg
\end{abstract}

\begin{abstract}
This article proposes an analytical method for decomposing a dynamic nonlinear system into a multiple model form in order to reduce its complexity and to study more easily identification, stability analysis and controller design problems. The majority of existing methods are order reduction based techniques, which come with an information loss of the initial system, whereas the method proposed here avoids this particular loss. The multiple model constitutes an efficient tool to represent nonlinear systems. These are decomposed into several linear time invariant systems (LTI) which are weighted and aggregated that allows to benefit from important analysis tools. This method is applied to a simplified activated sludge reactor model.
\end{abstract}

Keywords: dynamic modeling; reduction; weighting functions; subsystems; wastewater treatment

\section{INTRODUCTION}

The complexity problem of dynamic nonlinear systems appears in a great number of scientific and engineering domains. Some decomposition and simplification techniques were developed in the last years, in order to realize a complexity reduction, according to objectives like identification, controller design, stability analysis.

In most studies, the complexity reduction consists in the reduction of the system order that comes with an information loss (Dolgin and Zeheb [2005], Petzold and Zhu [1999], Sayesel and Barlas [2006]).

Another way to reduce the complexity of dynamic nonlinear systems is to rewrite the nonlinear system in a much easier way, by decomposing it into much simpler unities, without loosing information.

The multiple model (MM) representation (Murray-Smith and Johansen [1997]) constitutes actually a commonly used tool for nonlinear system modeling. The MM formalism is based on a decomposition of the system dynamic behavior into several operating zones, each zone being characterized by a submodel. Depending on the system evolution in each zone, each submodel contributes more or less to the approximation of the global system behavior. The contribution of each submodel in the global model, which is a convex combination of submodels, is defined by a weighting function. In the literature several equivalent terminologies are used to define this class of models: the MM (Murray-Smith and Johansen [1997]), the fuzzy Takagi-Sugeno model (Takagi and Sugeno [1985]), the polytopic linear model (PLM) (Angelis [2001]).

The convenience of using this type of models is that important properties like stability, controllability, observability, extensively used in the case of linear time-invariant sys- tems (LTI) can be generalized, at least partially, to MM if the constitutive submodels are linear and for particular weighting functions (Akhenak et al. [2004]).

The MM structure is then used here to reduce the complexity of a simplified ASM1 model (Activate Sludge Model no.1) (Olsson and Newell [1999]) which describes a biological degradation process of an activate sludge reactor. Different linearization techniques were proposed, like a linearization around one or several operating points (Smets et al. [2006]) in order to obtain a multiple model. The loss of information constitutes the first drawback of this technique. Secondly, the choice of premise variables expressing the nonlinearities of the system, just as the choice of different operating points, still remain very delicate.

Afterwards, we will present a systematic procedure to transform a nonlinear system by rewriting it into a MM form, avoiding the major drawbacks: the transformation is realized without loss of information, the choice of different operating points is no longer necessary and the choice of premise variables is realized in a more precise way. Starting with a general form of nonlinear system with bounded nonlinearities, a MM state representation is realized. This MM representation constitutes a quasi linear parameter varying (quasi-LPV) system because the convex combinations of constant matrices calculated from the polytope vertices give use to matrices with variable parameters. The vertices are obtained using the convex polytopic transformation (CPT) (Wang et al. [1996]). The constant matrices define the submodels and the nonlinearities are rejected into the submodel weighting functions. The MM obtained with this method is not unique, it depends on the choice of the scalars defining the CPT and on the quasi-LPV model structure. This method and its characteristics will be pointed out by accentuating on biological reactor model. 


\section{METHODOLOGY}

This section is dedicated to a general methodology to transform a nonlinear system presented in a general form into a multiple model. The proposed method is totally analytical and the obtained MM is equivalent to the initial nonlinear system i.e. the two representations give the same responses. This will be illustrated on a simplified activated sludge reactor model.

Considering a nonlinear system with bounded nonlinearities, the MM form is written. In general, for a nonlinear dynamic system, this representation is not unique, that is the reason why different criteria of choice for a MM form have to be defined to match the study objectives. To each MM form corresponds a premise variable set. Each premise variable will be partitioned in two parts using the convex polytopic transformation (Morère [2000]). The two partitions of each premise variable will contribute to the construction of the corresponding weighting functions of the MM. The multiple model will thus be a convex combination of linear submodels (Murray-Smith and Johansen [1997]), the nonlinearity being transferred into weighting functions related to each submodel.

\subsection{Analytical method of structure simplification}

The activated sludge reactor model, like a large category of nonlinear dynamic systems, can be represented by:

$$
\left\{\begin{array}{l}
\dot{x}(t)=f(x(t), u(t)) \\
y(t)=g(x(t), u(t))
\end{array}\right.
$$

where $f(x(\cdot), u(\cdot)) \in \mathbb{R}^{n}$ and $g(x(\cdot), u(\cdot)) \in \mathbb{R}^{l}$.

To reduce the complexity of nonlinear dynamic systems (1), a system decomposition into a multiple model form is realized. This type of model allows us to represent nonlinear dynamic systems into a convex combination of linear submodels:

$$
\left\{\begin{array}{l}
\dot{x}(t)=\sum_{i=1}^{r} \mu_{i}(x, u)\left[A_{i} x(t)+B_{i} u(t)\right] \\
y(t)=\sum_{i=1}^{r} \mu_{i}(x, u)\left[C_{i} x(t)+D_{i} u(t)\right]
\end{array}\right.
$$

$x \in \mathbb{R}^{n}$ is the state vector, $u \in \mathbb{R}^{m}$ is the input vector and $y \in \mathbb{R}^{l}$ the output vector. $A_{i}, B_{i}, C_{i}, D_{i}$ are constant matrices of appropriate dimensions. The function $\mu_{i}(x, u)$ represents the weight of the $i^{t h}$ submodel $\left\{\left(A_{i}, B_{i}, C_{i}, D_{i}\right\}\right.$. The weighting functions $\mu_{i}(x, u)$ have the following property:

$$
\sum_{i=1}^{r} \mu_{i}(x, u)=1 \text { and } \mu_{i}(x, u) \geq 0, \forall(x, u) \in \mathbb{R}^{n} \times \mathbb{R}^{m}
$$

In order to obtain the multiple model form, first the system will be transformed in a quasi-LPV form. This is an intermediate form which allows to obtain the multiple model structure. Under the hypothesis that $f(x(t), u(t))$ and $g(x(t), u(t))$ are continuous and bounded in $U \subseteq \mathbb{R}^{n}$ with $f(0, \cdot)=0$ and $g(0, \cdot)=0$, the system (1) can be represented in a quasi-LPV form:

$$
\left\{\begin{array}{l}
\dot{x}(t)=A(x(t), u(t)) x(t)+B(x(t), u(t)) u(t) \\
y(t)=C(x(t), u(t)) x(t)+D(x(t), u(t)) u(t)
\end{array}\right.
$$

where $A(x, u) \in \mathbb{R}^{n \times n}, B(x, u) \in \mathbb{R}^{n \times m}, C(x, u) \in \mathbb{R}^{l \times n}$, $D(x, u) \in \mathbb{R}^{l \times m}$.

Let us denote $a_{i, j}(x, u)$ (resp. $b_{i, j}(x, u), c_{i, j}(x, u)$ and $d_{i, j}(x, u)$ ) the entry of $A(x, u)$ (respectively $B(x, u)$, $C(x, u)$ and $D(x, u))$ lying on the $i^{t h}$ row and the $j^{\text {th }}$ column.

The matrix function $A(x, u)$ is in this case given by:

$$
A(x, u)=\left[\begin{array}{ccc}
a_{1,1}(x, u) & \cdots & a_{1, n}(x, u) \\
\vdots & & \\
a_{n, 1}(x, u) & \cdots & a_{n, n}(x, u)
\end{array}\right]
$$

$B(x, u), C(x, u)$ and $D(x, u)$ are constructed in the same way with appropriate dimensions.

Considering the quasi-LPV form resulting from system (1) and given in an explicit way in (3), we define the premise variable set $V_{z}$ in the following way:

$$
\begin{aligned}
V_{z} & =\left\{a_{i, q}(x, u) \mid a_{i, q} \neq \text { const }, i=\overline{1, n}, q=\overline{1, n}\right\} \\
& \cup\left\{b_{i, s}(x, u) \mid b_{i, s} \neq \text { const }, i=\overline{1, n}, s=\overline{1, m}\right\} \\
& \cup\left\{c_{j, q}(x, u) \mid c_{j, q} \neq \text { const }, j=\overline{1, l}, q=\overline{1, n}\right\} \\
& \cup\left\{d_{j, s}(x, u) \mid d_{j, s} \neq \text { const }, j=\overline{1, l}, s=\overline{1, m}\right\}
\end{aligned}
$$

where $\overline{1, n}=\{1, \ldots, n\}$ and when $a_{., .} \neq$const. means that $a$., does not reduce to a constant value. In fact every time varying entry of the matrices $A(x, u), B(x, u), C(x, u)$ and $D(x, u)$ will be considered as a decision variable, denoted $z_{i}(x, u)$.

Subsequently, this set $\left(V_{z}\right)$ is noted more simply:

$$
V_{z}=\left\{z_{1}(x, u), \ldots, z_{p}(x, u)\right\}
$$

where $p$ (verifying $p \leq(n+l)(n+m))$ denotes the number of premise variables and is the number of nonlinearities identified using the quasi-LPV form of the system (1). $z_{1}(x, u), \ldots, z_{p}(x, u)$ are the premise variables.

Remark 1. $(n+l) \times(n+m)$ represents the largest number of premise variables. However, for practical applications the number of premise variables is less that this bound.

In general, the quasi-LPV form (3) for a nonlinear system of the form (1) is not unique; for each quasi-LPV form exist a particular premise variable set; choosing a quasiLPV form is equivalent to choose the premise variable set. The choice of the premise variable set $V_{z}$ is important, because it has an influence on the submodel numbers and on the global model structure. This is a degree of freedom that should be used to ease the controllability, the observability and the stability analysis studies. First of all, the controllability/observability of each submodel is necessary to ensure the controllability/observability of the global system, represented in a multiple model form (Guerra et al. [2008]). Thus, the quasi-LPV forms producing submodels which are not controllable/observable will not be considered. However it should be pointed out that this is not a sufficient condition to ensure the controllability/observability of the multiple model (Murphey and Burdick [2002]). Secondly, in the stability analysis study of multiple model, different techniques are proposed to reduce the number of LMI (Linear Matrix Inequality) conditions, which extend the solution existence (Bergsten et al. [2002]) and leads to less computational requirement (Tanaka et al. [2007]). Due to the convexity of the interpolation made between the submodels, the LMI are verified 
for all the vertices. As a consequence, the number of LMI to be satisfied is linear or polynomial in the number of submodels (Tanaka and Wang [2001]). That is the reason why it is tried to find a multiple model composed by a minimal number of submodels. This number is related to the number of premise variables, so that a quasi-LPV form which has a set of minimal number of premise variables will be preferred. Besides that, the complexity of the LMI conditions increases with the number of state variables involved. In order to ensure the solution existence, the dimension of the state vector should be made as small as possible (Bergsten et al. [2002]). Taking into account the previous remarks, the following rules can guide the choice of the quasi-LPV form leading to the simplest model:

- Eliminate all quasi-LPV forms for which the matrices have null columns $(B)$ and/or rows $(C)$.

- Identify the decompositions that contain common premise variables for different state and/or output equations (3), reducing in this way the number of premise variables.

- Among quasi-LPV forms for which the premise variables $z_{i}(x, u)(i=1, \ldots, p)$ depend on the same number of state variables, choose the quasi-LPV form that contains the minimal number of premise variables $z_{i}(x, u)$ (taking into account the next rule).

- Among quasi-LPV forms that have the same number of premise variables $z_{i}(x, u) \quad(i=1, \ldots, p)$, choose the quasi-LPV form for which the premise variables $z_{i}(x, u)$ depend on a minimal number of state variables.

Let us introduce a notion used here after: the convex polytopic transformation.

Lemma 1. Let $h(x(t), u(t))$ be a bounded and continuous function from $\left[x_{0}, x_{1}\right] \times\left[u_{0}, u_{1}\right]$ to $\boldsymbol{R}$, with $x_{0}, x_{1} \in \boldsymbol{R}^{n}$, $u_{0}, u_{1} \in \boldsymbol{R}^{m}$. Then there exist two functions

$$
\begin{gathered}
F_{i}:\left[x_{0}, x_{1}\right] \times\left[u_{0}, u_{1}\right] \longmapsto[0,1](i=1,2) \\
(x(t), u(t)) \longmapsto F_{i}(x(t), u(t))
\end{gathered}
$$

with $F_{1}(x(t), u(t))+F_{2}(x(t), u(t))=1$ such as:

$$
h(x(t), u(t))=F_{1}(x(t), u(t)) \cdot h_{1}+F_{2}(x(t), u(t)) \cdot h_{2}
$$

for all $h_{1} \geq \max _{x, u}\{h(x, u)\}$ and $h_{2} \leq \min _{x, u}\{h(x, u)\}$

The two functions $F_{1}$ and $F_{2}$ are defined as:

$$
\begin{aligned}
& F_{1}(x(t), u(t))=\frac{h(x(t), u(t))-h_{2}}{h_{1}-h_{2}} \\
& F_{2}(x(t), u(t))=\frac{h_{1}-h(x(t), u(t))}{h_{1}-h_{2}}
\end{aligned}
$$

Let us note that this decomposition is not unique.

After the choice of a quasi-LPV form and of the premise variables (using the rules mentioned above), a convex polytopic transformation for the $p$ premise variables is performed.

$$
\begin{gathered}
z_{j}(x, u)=F_{j, 1}\left(z_{j}\right) \cdot z_{j, 1}+F_{j, 2}\left(z_{j}\right) \cdot z_{j, 2} \\
F_{j, 1}\left(z_{1}\right)=\frac{z_{j}(x, u)-z_{j, 2}}{z_{j, 1}-z_{j, 2}} \\
F_{j, 2}\left(z_{1}\right)=\frac{z_{j, 1}-z_{j}(x, u)}{z_{j, 1}-z_{j, 2}}
\end{gathered}
$$

$$
\begin{aligned}
& z_{j, 1}=\max _{x, u}\left\{z_{j}(x, u)\right\} \\
& z_{j, 2}=\min _{x, u}\left\{z_{j}(x, u)\right\}
\end{aligned}
$$

Using the convex polytopic transformation (Lemma 1), we generate the $2^{p}$ submodels of a MM characterized by $p$ premise variables. Each premise variable is thus described like in (5) with the partitions (6), and the scalars $z_{j, 1}$ and $z_{j, 2}$ defined in $(7)$, for all $j=1, \ldots, p$.

To each submodel $i$ corresponds a $p$-uplet $\sigma_{i}$ which codes the partitions of the premise variables occuring in the corresponding weighting function, just like in table 1 , where the case of three premise variables is illustrated. Considering the $i^{\text {th }}$ row, the entries equal to 1 indicate the partition of each premise variable, intervening in the $i^{\text {th }}$ weighting function.. Multiplying the functions describing these partitions, we obtain the corresponding weighting function $\mu_{i}(z)$ :

$$
\mu_{i}(z)=\mu_{i}(x, u)=\prod_{k=1}^{p} F_{k, \sigma_{i}^{k}}\left(z_{k}(x, u)\right)
$$

where $\sigma_{i}^{k}$ represents the index in the $k^{t h}$ position in the $p$-uplet $\sigma_{i}$. Then $\sum_{i=1}^{r} \mu_{i}(z)=1$ and $\mu_{i}(z) \geq 0(i=1, \ldots, r)$, since:

$$
F_{j, 1}\left(z_{j}(x, u)\right)+F_{j, 2}\left(z_{j}(x, u)\right)=1, \forall j=1, \ldots, p
$$

Remark 2. The number of submodels that constitute the multiple model is $r=2^{p} \leq 2^{(n+l)(n+m)}$.

The matrices with variable parameters intervening in the quasi-LPV form of system (1) are linear combinations of constant matrices $\left(\mathcal{A}_{j}, \mathcal{B}_{j}, \mathcal{C}_{j}, \mathcal{D}_{j}\right)$; for example, the matrix $A(x, u)$ may be expressed in the following way:

$$
A(x, u)=\mathcal{A}_{0}+\sum_{j \in \mathcal{I}_{A}} z_{j}(x, u) \cdot \mathcal{A}_{j}
$$

where the set $\mathcal{I}_{A}$ contains all the indexes corresponding to the premise variables which are contained in the entries of the matrix $A(x, u)$. The matrix $\mathcal{A}_{0}$ contains the constant entries $\left(a_{i, j}\right)$ of the matrix $A(x, u)$. The matrix $\mathcal{A}_{j}$ contains at the position corresponding to $z_{j}$ the constant term 1 , and zero for all the other positions.

In the matrices $A(x, u), B(x, u), C(x, u)$ and $D(x, u)$, written like in $(9)$, call upon the premise variables $z_{j}$ for $j=1, \ldots, p$; in this way, the matrices $A, B, C$ and $D$ will be evaluated at the vertices of the polytope defined by the premise variable partitions that intervene in these matrices for determining the matrices $A_{i}, B_{i}, C_{i}$ and $D_{i}(i=1, \ldots, r)$, that are constant coefficients matrices relatives to each submodel:

$$
A_{i}=\mathcal{A}_{0}+\sum_{j \in \mathcal{I}_{A}} z_{j, \sigma_{i}^{j}} \cdot \mathcal{A}_{j}
$$

$B_{i}, C_{i}$ and $D_{i}$ can be written in the same way.

We can give the following result:

The multiple model (2) which represents a convex combination of linear submodels $\left\{\left(A_{i}, B_{i}, C_{i}, D_{i}\right)\right\}(i=1, \ldots, r)$ is equivalent to the system (1).

For reasons of space the proof of this theorem can not be 
developed here.

We can notice that obtaining a MM with this method generally needs neither to make some approximation nor to choose linearization points.

This method is illustrated in the next session with a biological reactor model.

\section{THE ACTIVATED SLUDGE REACTOR MODELING}

\subsection{The biological reactor model}

In this section, some general considerations are given concerning an activated sludge reactor model, the reduced ASM1 model: the carbonated pollution part of ASM1 model will be considered.

The activated sludge wastewater treatment consists in mixing used waters with a rich mixture of bacteria in order to degrade the organic matter. In the activated sludge reactor study, several operating modes can be considered: reactor with constant volume, regulation from a reference volume, free flow at the output reactor, controllable input and output flows. The first situation is reported here.

The elimination process of carbonated pollution is realized in the bioreactor-clarifier group. The simplified diagram is given in figure 1. The reactor-clarifier group can be represented by the following nonlinear system:

$$
\left\{\begin{array}{c}
\frac{d V(t)}{d t}=q_{\text {in }}(t)+q_{R}(t)-q_{\text {out }}(t) \\
\frac{d\left(V(t) \cdot X_{B H}(t)\right)}{d t}=q_{\text {in }}(t) X_{B H, \text { in }}(t)+q_{R}(t) X_{B H, R}(t) \\
-q_{\text {out }}(t) X_{B H, \text { out }}(t)+r_{H}(t) V(t) \\
\frac{d\left(V(t) \cdot S_{S}(t)\right)}{d t}=q_{\text {in }}(t) S_{S, \text { in }}(t)+q_{R}(t) S_{S, R}(t) \\
-q_{\text {out }}(t) S_{S, \text { out }}(t)+r_{S}(t) V(t) \\
\frac{d\left(V(t) \cdot S_{O}(t)\right)}{d t}=q_{\text {in }}(t) S_{O, \text { in }}(t)+q_{R}(t) S_{O, R}(t) \\
+K q_{a}(t) V(t)\left(S_{O, \text { sat }}-S_{O}(t)\right)
\end{array}\right.
$$

where the variables involved are: $V$ the reactor volume, $X_{B H}$ the heterotrophic biomass concentration, $X_{B H, R}$ the recycled one, $S_{S}$ the fast biodegradable substrate concentration, $S_{O}$ the dissolved oxygen concentration, $q$ the flow and $q_{a}$ the air flow. The in and out indexes correspond respectively to the reactor input and output, $q_{R}, q_{W}$ are respectively the recycled and the rejected flow. $r_{H}, r_{S}$ and $r_{O}$ representing the kinetics of heterotrophic biomass $X_{B H}$, carbon $S_{S}$ and oxygen $S_{O}$ reactions in a heterotroph and aerobic environment are modeled by:

$$
\left\{\begin{aligned}
r_{H}= & \mu_{H} \frac{S_{S}}{K_{S}+S_{S}} \frac{S_{O}}{K_{O H}+S_{O}} X_{B H}-b_{H} X_{B H} \\
r_{S}= & -\frac{1}{Y_{H}} \mu_{H} \frac{S_{S}}{K_{S}+S_{S}} \frac{S_{O}}{K_{O H}+S_{O}} X_{B H} \\
& +(1-f) b_{H} X_{B H} \\
r_{O}= & \frac{Y_{H}-1}{Y_{H}} \mu_{H} \frac{S_{S}}{K_{S}+S_{S}} \frac{S_{O}}{K_{O H}+S_{O}} X_{B H}
\end{aligned}\right.
$$

The time dependence of different variables will not be explicitly mentioned in the future equations, in order to facilitate the writing.

The different coefficients intervening in (11) and (12) are: $S_{O, \text { sat }}=10\left[\mathrm{~g} / \mathrm{m}^{3}\right]$ (oxygen saturation concentration), $K_{S}=20\left[\mathrm{~g} / \mathrm{m}^{3}\right]$ and $K_{O H}=0.2\left[\mathrm{~g} / \mathrm{m}^{3}\right]$ (half-saturation constants), $Y_{H}=0.6$ (g cell COD formed) and $f=0.1$ (conversion rate coefficients for the products in different reactional processes (increase or mortality)), $b_{H}=$ $0.4[1 / 24 h]$ and $\mu_{H}=3.733[1 / 24 h]$ (heterotrophic kinetic coefficients), $K=2.3\left[1 / \mathrm{m}^{3}\right]$ (regulator gain in oxygen). The clarifier is supposed to be perfect with no internal dynamic process and no biomass in the effluent. In this case, we can write:

$$
\begin{aligned}
& \left(q_{i n}+q_{R}\right) X_{B H}=\left(q_{R}+q_{W}\right) X_{B H, R} \\
& S_{S, R}=S_{S}
\end{aligned}
$$

Under the reactor homogeneity hypothesis, the following assumptions are made:

$$
\left\{\begin{array}{l}
X_{B H, \text { out }}=X_{B H} \\
S_{S, \text { out }}=S_{S} \\
S_{O, \text { out }}=S_{O}
\end{array}\right.
$$

It is supposed that the dissolved oxygen concentration at the reactor input $\left(S_{O, \text { in }}\right)$ is null.

In general, $q_{R}$ and $q_{W}$ represent fractions of input flow $q_{i n}$ :

$$
\begin{array}{cc}
q_{R}=f_{R} q_{i n}, & 1 \leq f_{R} \leq 2 \\
q_{W}=f_{W} q_{i n}, & 0<f_{W}<1
\end{array}
$$

The reactor volume is assumed to be constant and thus: $q_{\text {out }}=q_{\text {in }}+q_{R}$. Replacing $r_{H}, r_{S}$ and $r_{O}$ given in (12) and taking into account the above assumptions (by eliminating the first equation which corresponds to the volume variation) then the system (11) becomes:

$$
\left\{\begin{array}{c}
\dot{X}_{B H}=\frac{q_{i n}}{V} X_{B H, i n}-\frac{q_{W}}{V} \frac{q_{i n}+q_{R}}{q_{W}+q_{R}} X_{B H} \\
\quad+\mu_{H} \frac{S_{S}}{K_{S}+S_{S}} \frac{S_{O}}{K_{O H}+S_{O}} X_{B H}-b_{H} X_{B H} \\
\dot{S}_{S}=\frac{q_{i n}}{V}\left(S_{S, i n}-S_{S}\right)+(1-f) b_{H} X_{B H} \\
\quad-\frac{\mu_{H}}{Y_{H}} \frac{S_{S}}{K_{S}+S_{S}} \frac{S_{O}}{K_{O H}+S_{O}} X_{B H} \\
\dot{S}_{O}=-\frac{q_{i n}}{V} S_{O}+K q_{a}\left(S_{O, s a t}-S_{O}\right) \\
+\frac{Y_{H}-1}{Y_{H}} \mu_{H} \frac{S_{S}}{K_{S}+S_{S}} \frac{S_{O}}{K_{O H}+S_{O}} X_{B H}
\end{array}\right.
$$

\subsection{The multiple model for the biological reactor}

To obtain the multiple model form, in a first step, the system (14) has to be transformed into a quasi-LPV form, just as in (3). Taking into account the rules mentioned in the previous section, the chosen quasi-LPV form is represented by:

$$
\begin{array}{ll}
A\left(z_{1}, z_{2}, z_{3}\right)=\left(\begin{array}{ccc}
a_{1,1} & 0 & 0 \\
a_{2,1} & a_{2,2} & 0 \\
a_{3,1} & 0 & a_{3,3}
\end{array}\right) & x=\left(\begin{array}{c}
X_{B H} \\
S_{S} \\
S_{O}
\end{array}\right) \\
B\left(z_{2}\right)=\left(\begin{array}{ccc}
b_{1,1} & 0 & 0 \\
0 & b_{2,2} & 0 \\
0 & 0 & b_{3,3}
\end{array}\right) & u=\left(\begin{array}{c}
X_{B H, i n} \\
S_{S, i n} \\
q_{a}
\end{array}\right)
\end{array}
$$


where

$$
\begin{aligned}
a_{1,1} & =\mu_{H} \cdot z_{1}-\frac{f_{W}\left(1+f_{R}\right)}{f_{W}+f_{R}} \cdot z_{2}-b_{H} \\
a_{2,1} & =-\frac{1}{Y_{H}} \mu_{H} \cdot z_{1}+(1-f) b_{H} \\
a_{2,2} & =-z_{2} \\
a_{3,1} & =\frac{Y_{H}-1}{Y_{H}} \mu_{H} \cdot z_{1} \\
a_{3,3} & =-K \cdot z_{3}-z_{2} \\
b_{1,1} & =b_{2,2}=z_{2} \\
b_{3,3} & =K \cdot S_{O, \text { sat }}
\end{aligned}
$$

The system presents three nonlinearities and we have chosen as premise variables:

$$
\left\{\begin{array}{l}
z_{1}\left(S_{S}, S_{O}\right)=\frac{S_{S}}{K_{S}+S_{S}} \cdot \frac{S_{O}}{K_{O H}+S_{O}} \\
z_{2}\left(q_{i n}, V\right)=\frac{q_{i n}}{V} \\
z_{3}\left(q_{a}\right)=q_{a}
\end{array}\right.
$$

Remark. The flow $q_{i n}$ is an input for the biological reactor and is not integrated in the quasi-LPV form (15) because this will generate a null column in the matrix $B$, which is not a desired structure for controllability studies.

In a second step, using the transformation presented in Lemma 1, the three premise variables $z_{1}, z_{2}$ and $z_{3}$ are written like in (5), where each premise variable is constructed as a convex sum $(6)$ of scalars $(7)(j=1, \ldots, 3)$. The maxima and the minima in (7) are calculated using the fact that $V, S_{S}, S_{O}, q_{a}$ and $q_{i n}$ are bounded.

In table 1 , are generated all the submodels of a multiple model characterized by three premise variables $z_{j}(x, u)$ $(j=1, \ldots, 3)$ which are partitioned into two parts, the number of submodels being equal to $2^{3}$.

To each submodel $i$ corresponds a triplet $\sigma_{i}$ which codes the partitions of the premise variables $z_{j}(x, u)(j=1, \ldots, 3)$ intervening in the corresponding weighting function. In this way, in the table 1 , considering the $i^{\text {th }}$ row, the entries equal to 1 indicate the partition of each premise variable, intervening in the weighting function $\mu_{i}(x)$ :

$$
\begin{aligned}
& \mu_{1}(x)=F_{1,1}\left(z_{1}\right) F_{2,1}\left(z_{2}\right) F_{3,1}\left(z_{3}\right) \\
& \mu_{2}(x)=F_{1,1}\left(z_{1}\right) F_{2,1}\left(z_{2}\right) F_{3,2}\left(z_{3}\right) \\
& \mu_{3}(x)=F_{1,1}\left(z_{1}\right) F_{2,2}\left(z_{2}\right) F_{3,1}\left(z_{3}\right) \\
& \mu_{4}(x)=F_{1,1}\left(z_{1}\right) F_{2,2}\left(z_{2}\right) F_{3,2}\left(z_{3}\right) \\
& \mu_{5}(x)=F_{1,2}\left(z_{1}\right) F_{2,1}\left(z_{2}\right) F_{3,1}\left(z_{3}\right) \\
& \mu_{6}(x)=F_{1,2}\left(z_{1}\right) F_{2,1}\left(z_{2}\right) F_{3,2}\left(z_{3}\right) \\
& \mu_{7}(x)=F_{1,2}\left(z_{1}\right) F_{2,2}\left(z_{2}\right) F_{3,1}\left(z_{3}\right) \\
& \mu_{8}(x)=F_{1,2}\left(z_{1}\right) F_{2,2}\left(z_{2}\right) F_{3,2}\left(z_{3}\right)
\end{aligned}
$$

To express the constant coefficient matrices $A_{i}$ and $B_{i}$ of (2), characterizing each submodel $i(i=1, \ldots, 8)$ we use the matrices defined in (15). Let us note that the premise variables $z_{1}, z_{2}$ and $z_{3}$ (resp. $z_{2}$ ) are involved in $A(x, u)$ (resp. $B(x, u)$ ). In this way, we will evaluate the matrices $A$ and $B$ at the vertices of the polytope defined by the partitions of premise variables which occur in these matrices. For example, to determine the matrices $A_{3}$ and $B_{3}$, we proceed in the following way. The triplet corresponding to the third line is retained $\sigma_{3}=(1,2,1)$, which has as components $\sigma_{3}^{k}(k=1, \ldots, 3)$ that can take the values 1 or 2 . Taking into account (15), (16) and (17), $A_{3}=A\left(z_{1, \sigma_{3}^{1}}, z_{2, \sigma_{3}^{2}}, z_{3, \sigma_{3}^{3}}\right)$ and $B_{3}=B\left(z_{2, \sigma_{3}^{2}}\right)$.

In a more general way $A_{i}$ and $B_{i}(i=1, \ldots, 8)$ are noted:

$$
\begin{aligned}
& A_{i}=A\left(z_{1, \sigma_{i}^{1}}, z_{2, \sigma_{i}^{2}}, z_{3, \sigma_{i}^{3}}\right) \\
& B_{i}=B\left(z_{2, \sigma_{i}^{2}}\right)
\end{aligned}
$$

The multiple model is obtained by an interpolation of the eight previous submodels, just as in $(2)(r=8)$. In figure 2 we can see the evolution of the system (14) (represented in the form (2)) and the weighting functions of the multiple model obtained for $f_{R}=1.0, f_{W}=0.037$, with the initial conditions: $\left(V(0), X_{B H}(0), S_{S}(0), S_{O}(0)\right)^{T}=$ $(1250,887,4.1,1.0)^{T}$. The volume is expressed in $m^{3}$. For space and clarity reasons only four weighting functions are presented in the figure $\left(\mu_{5}, \mu_{6}, \mu_{7}, \mu_{8}\right)$. It has to be pointed out that the evolution of the weighting functions depends on the control input evolution.

\section{CONCLUSION}

This article proposes a general analytical method to simplify the complexity of a nonlinear system. This substitution consists in finding a set of submodels with a simple linear structure and a set of appropriated weighting functions in order to combine these submodels to constitute the global model.

Let us insist to the fact that in order to obtain a multiple model, different techniques of linearization applied around different well chosen operating points were proposed in the literature. The major drawback is that these techniques imply a loss of information. Besides, the difficulty of choosing these different operating points is the reason why this article proposes a more general method to rewrite the nonlinear model into a multiple model form avoiding the need to choose these operating points and avoiding loss of information.

From the expression of a nonlinear system, we showed how to realize a systematic decomposition of a system into simpler linear systems by constructing a multiple model. It is important to note that the obtained multiple model has the same response that those of the initial system. Another important aspect concerning this decomposition method is the given possibility to reduce the number of submodels by an adequate choice of premise variables between several sets of candidate premise variables.

This method is applied to a simplified activated sludge reactor model and leads to a multiple model structure based on eight submodels. Even if in this article we have treated the case of a reduced ASM1 model, considering only the carbon pollution, the proposed method can be expanded to the complete ASM1 model, the difficulties being similar.

\section{ACKNOWLEDGMENT}

We acknowledge the financial support received from the "Fonds National de la Recherche Luxembourg". This research is also partially supported by the TASSILI no.07 program under MDU grant 714 .

\section{REFERENCES}

A. Akhenak, M. Chadli, J. Ragot, and D. Maquin. Estimation of state and unknown inputs of a nonlinear system 
represented by a multiple model. In 11th IFAC Symposium on Automation in Mining, Mineral and Metal processing, MMM, 2004.

G. Z. Angelis. System Analysis, Modelling and Control with Polytopic Linear Models. Technische Universiteit Eindhoven, 2001. URL http://alexandria.tue.nl/extra2/200110616.pdf.

P. Bergsten, R. Palm, and D. Driankov. Observers for Takagi-Sugeno fuzzy systems. IEEE Transactions on Systems, Man, and Cybernetics, Part B, 32:114-121, 2002.

Y. Dolgin and E. Zeheb. Model reduction of uncertain systems retaining the uncertainty structure. Systems and Control Letters, 54:771-779, 2005.

T.M. Guerra, A. Kruszewski, and M. Bernal. Control law proposition for the stabilisation of discrete takagisugeno models. IEEE Transactions on Fuzzy Systems (Accepted for future publication. Date published Online: 2008-07-16), 2008.

Y. Morère. Mise en oeuvre de lois de commandes pour les modèles flous de type Takagi-Sugeno. $\mathrm{PhD}$ thesis, Université de Valenciennes et du Haut Cambrésis, Lille, France, 2000.

T.D. Murphey and J.W. Burdick. Nonsmooth controllability theory and an example. In Proceedings of the 41 st IEEE Conference on Decision and Control, volume 1, pages 370-376, 2002. 10-13 December 2002.

R. Murray-Smith and T.A. Johansen. Multiple model approaches to modelling and control. Taylor \& Francis, London, 1997.

G. Olsson and B. Newell. Wastewater Treatment Systems. Modelling, Diagnosis and Control. IWA Publishing, 1999.

L. Petzold and W. Zhu. Model reduction for chemical kinetics: an optimization ppproach. American Institute of Chemical Engineers Journal, 45(4):869-886, 1999.

A. K. Sayesel and Y. Barlas. Model simplification and validation with indirect structure validity tests. System Dynamics Reviews, 22(3):241-262, 2006.

I.Y. Smets, L. Verdickt, and J. Van Impe. A linear ASM1 based multi-model for activated sludge systems. Mathematical and Computer Modelling of Dynamical Systems, 12(5):489-503, 2006. doi: 10.1080/13873950600723467.

T. Takagi and M. Sugeno. Fuzzy identification of systems and its application to modelling and control. IEEE Transactions on Systems, Man and Cybernetic, 15:166$172,1985$.

K. Tanaka and H.O. Wang. Fuzzy Control System Design and analysis. A Linear Matrix Inequality Approach. John Wiley \& Sons Inc., 2001.

K. Tanaka, H. Ohtake, and H.O. Wang. A descriptor system approach to fuzzy control system design via fuzzy lyapunov functions. IEEE Transactions on Fuzzy Systems, 15:333-341, 2007.

H. O. Wang, K. Tanaka, and M. Griffin. An approach to fuzzy control of nonlinear systems: stability and design issues. IEEE Transactions on Fuzzy Systems, 4(1):14$23,1996$.

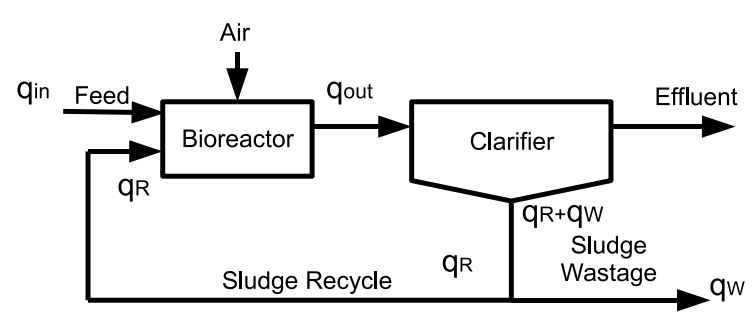

Fig. 1. The diagram of activated sludge wastewater treatment process

\begin{tabular}{|c|c|c|c|c|c|c|c|}
\hline \multirow{3}{*}{$\begin{array}{c}\text { Model } \\
i\end{array}$} & \multicolumn{6}{|c|}{ Partitions } & \multirow{3}{*}{$\sigma_{i}$} \\
\hline & \multicolumn{2}{|c|}{$z_{1}$} & \multicolumn{2}{|c|}{$z_{2}$} & \multicolumn{2}{|c|}{$z_{3}$} & \\
\hline & $F_{1,1}$ & $F_{1,2}$ & $F_{2,1}$ & $F_{2,2}$ & $F_{3,1}$ & $F_{3,2}$ & \\
\hline 1 & 1 & 0 & 1 & 0 & 1 & 0 & $(1,1,1)$ \\
\hline 2 & 1 & 0 & 1 & 0 & 0 & 1 & $(1,1,2)$ \\
\hline 3 & 1 & 0 & 0 & 1 & 1 & 0 & $(1,2,1)$ \\
\hline 4 & 1 & 0 & 0 & 1 & 0 & 1 & $(1,2,2)$ \\
\hline 5 & 0 & 1 & 1 & 0 & 1 & 0 & $(2,1,1)$ \\
\hline 6 & 0 & 1 & 1 & 0 & 0 & 1 & $(2,1,2)$ \\
\hline 7 & 0 & 1 & 0 & 1 & 1 & 0 & $(2,2,1)$ \\
\hline 8 & 0 & 1 & 0 & 1 & 0 & 1 & $(2,2,2)$ \\
\hline
\end{tabular}

Table 1. Decomposition table for a multiple model with three premise variables and two partitions for each variable

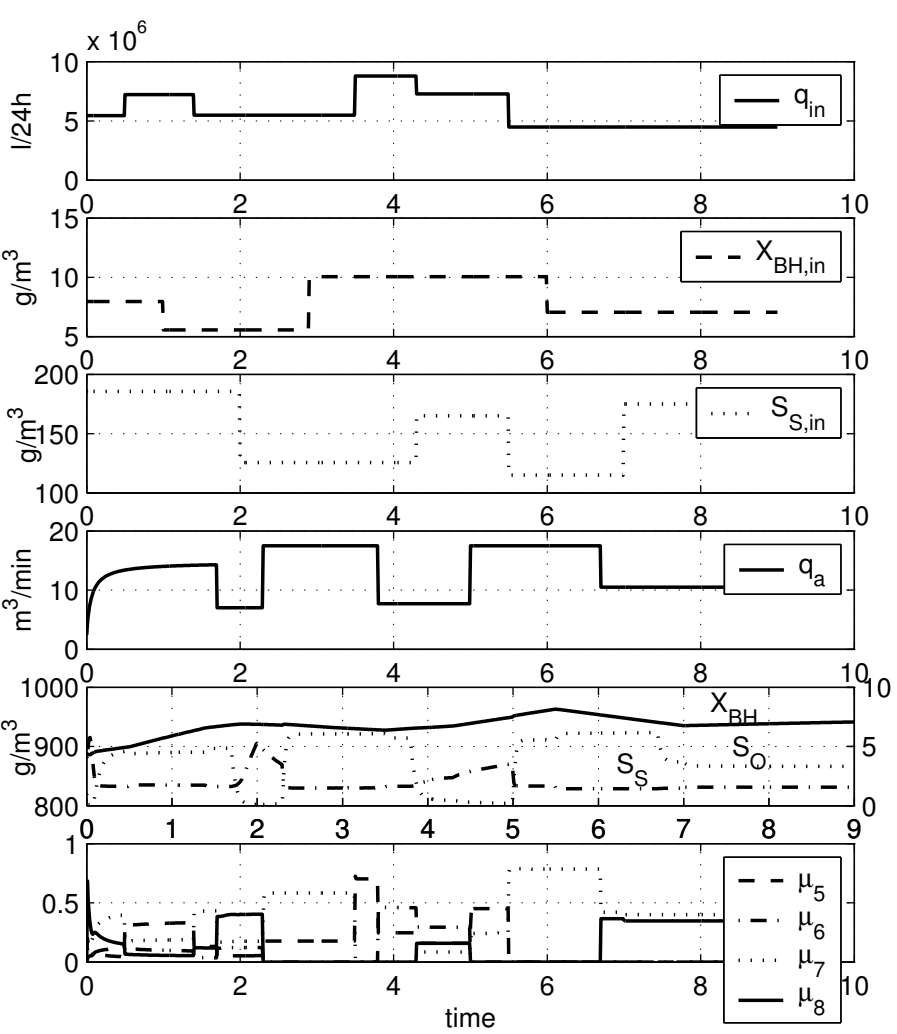

Fig. 2. The evolution of system (14) presented into a multiple model form and some weighting functions of the multiple model 